\section{OP0202 EFFECT OF RSLV-132 ON FATIGUE IN PATIENTS WITH PRIMARY SJÖGREN'S SYNDROME - RESULTS OF A PHASE II RANDOMISED, DOUBLE-BLIND, PLACEBO-CONTROLLED, PROOF OF CONCEPT STUDY}

Benjamin Fisher ${ }^{1}$, Francesca Barone ${ }^{1}$, Kerry Jobling ${ }^{2}$, Peter Gallagher ${ }^{3}$, Victoria Macrae ${ }^{2}$, Andrew Filby ${ }^{4}$, Gillian Hulmes ${ }^{4}$, Paul Milne ${ }^{5}$,

Emmanuella Traianos ${ }^{5}$, Valentina lannizzotto@bham.Ac.uk ${ }^{1}$, Alexandre Dumusc ${ }^{1}$, Simon J. Bowman ${ }^{6}$, Jessica Tarn ${ }^{5}$, Dennis Lendrem ${ }^{5}$, Daniel Burge ${ }^{7}$, James Posada ${ }^{7}$, Wan-fai $\mathrm{Ng}^{2,5}$. ${ }^{1}$ Birmingham University, Birmingham, United Kingdom; ${ }^{2}$ Newcastle upon Tyne Hospitals NHS Foundation Trust, Newcastle upon Tyne, United Kingdom; ${ }^{3}$ Newcastle University, Institute of Neuroscience, Newcastle upon Tyne, United Kingdom; ${ }^{4}$ Newcastle University, Newcastle upon Tyne, United Kingdom; ${ }^{5}$ Newcastle University, Institute of Cellular Medicine, Newcastle upon Tyne, United Kingdom; ${ }^{6}$ University Hospitals Birmingham, Birmingham, United Kingdom; ${ }^{7}$ Resolves Therapeutics, Seattle, United States of America

Background: Fatigue is the key symptom that leads to poor health related quality of life and loss of work productivity in patients with primary Sjogren's syndrome (pSS). RSLV-132 is a first-in-class drug comprising RNase1 fused to the Fc region of IgG1. It is designed to increase serum RNase activity to digest RNA associated immune complexes, thereby reducing Toll-like receptor (TLR) activation and subsequent production of type 1 interferon (IFN), B-cell proliferation, and autoantibody production - mechanisms that are key to pSS pathogenesis. IFN pathway dysregulation has also been implicated in fatigue.

Objectives: To explore the clinical efficacy of RSLV-132 in improving patientreported outcomes (PRO), particularly fatigue among patients with pSS.

Methods: PSS patients with positive anti-Ro and IFN gene expression signature were randomised $3: 1$ to RSLV-132 $10 \mathrm{mg} / \mathrm{kg}$ IV or placebo (PBO) at weeks $0,1,2$, and then fortnightly until week 12 . Use of hydroxycholoroquine, other immunomodulatory therapies or prednisolone $>10 \mathrm{mg}$ daily were not permitted. There was no minimum entry criteria for EULAR Sjogren's syndrome disease activity (ESSDAI) or EULAR Sjogren's syndrome patient reported index (ESSPRI). The primary endpoint was changes in gene or protein expression levels in blood indicative of reduced inflammation. Secondary endpoints, among others, included safety and tolerability, and changes in PRO between baseline and week 14 including ESSPRI, FACIT, Fatigue visual analogue score (0-100), Profile of fatigue (PRO-F (0-7)) and Neuropsychological tests.

Results: Thirty patients were randomised $(R S L V=22, P B O=8)$. Baseline clinical and demographic characteristics were comparable between groups. Two subjects randomised to active drug withdrew from the study before dosing.

Among the patients receiving RSLV-132, the mental component of PRO-F improved by 1.53 points compared to worsening of 0.06 points in the PBO group $(p=0.046)$. Consistently, there was a significant improvement in the RSLV-132 group in their Digital Symbol Substitution Test performance with a reduction of $16.4 \mathrm{~s}$ in completing the test compared to an increase of $2.8 \mathrm{~s}$ in the PBO group $(p=0.024)$. The physical component of PRO-F improved by 0.8 points in the RSLV-132 group compared to improvement of 0.06 points in the PBO group $(p=0.142)$. Similar trends were observed for ESSPRI and FACIT-F scores.

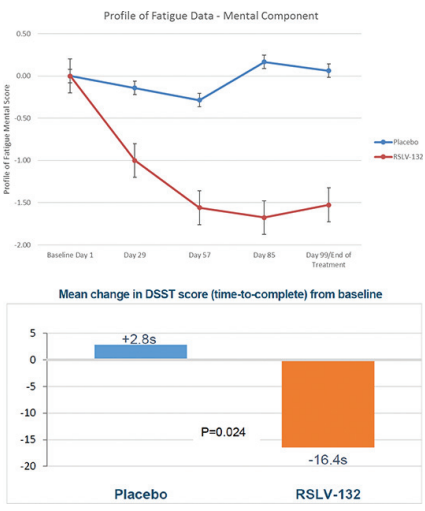

There was no drug effect on ESSDAI but the baseline median ESSDAI scores were low for both groups $(\mathrm{RSLV}=3(\operatorname{IQR}(0,11), \mathrm{PBO}=5(\operatorname{IQR}(0,18))$.

Treatment Emergent Adverse Events were reported by all participants and similar between arms; overall RSLV-132 was safe and well-tolerated. One SAE of parotitis in the RSLV-132 arm occurred 88 days after last dose of study drug and was considered unrelated to the RSLV-132.

Analyses of the primary endpoints and other secondary endpoints are ongoing. Conclusion: RSLV-132 is a promising therapy to improve the symptoms of fatigue in patients with pSS, with a good safety profile. Further investigation of its use in pSS is warranted.

\section{REFERENCE:}

[1] Study supported by Resolve Therapeutics

Disclosure of Interests: Benjamin Fisher Consultant for: Novartis, Roche, Medlmmune and BMS, Francesca Barone Grant/research support from: GlaxoSmithKline, Roche, UCB Pharma, Actelion, ONO Pharmaceutical, Consultant for: GlaxoSmithKline, Roche, Actelion, ONO Pharmaceutical, Kerry Jobling: None declared, Peter Gallagher: None declared, Victoria Macrae: None declared, Andrew Filby: None declared, Gillian Hulmes: None declared, Paul Milne: None declared, Emmanuella Traianos: None declared, Valentina lannizzotto@bham. ac.uk: None declared, Alexandre Dumusc: None declared, Simon J. Bowman Grant/research support from: Previously

UCB Pharma (to University of Birmingham) and Roche, Consultant for: 2016-7: Novartis, Mitsubishi Tanabe Pharma

2017-8: AstraZeneca, Medlmmune, GFK, Xtlbio, ONO Pharmaceutical

2018-9: Novartis, AstraZeneca, UCB Pharma, Jessica Tarn: None declared, Dennis Lendrem: None declared, Daniel Burge Employee of: Resolve Therapeutics, Medical Monitor for the clinical trial, James Posada Shareholder of: Owns equity of Resolve Therapeutics, Employee of: Founder and CEO of Resolve Therapeutics, Wan-fai Ng Grant/research support from: I have research collaborations with Resolve Therapeutics, electroCore, GSK, and Abbvie., Consultant for: Novartis, GSK, Abbvie, Medlmmune, Pfizer, BMS

DOI: 10.1136/annrheumdis-2019-eular.3098

\section{THURSDAY, 13 JUNE 2019}

\section{Molecular fingerprinting}

\section{OP0203 \\ CHARACTERIZING THE EPIGENOMIC LANDSCAPE OF PSORIASIS PATIENTS DESTINED TO DEVELOP} PSORIATIC ARTHRITIS

Remy Pollock ${ }^{1}$, Rohan Machhar ${ }^{1}$, Vinod Chandran ${ }^{1,2,3,4}$, Dafna D Gladman ${ }^{1,2}$. ${ }^{1}$ Krembil Research Institute, University Health Network, Psoriatic Disease Research Program, Toronto, Canada; ${ }^{2}$ Faculty of Medicine, University of Toronto, Division of Rheumatology, Toronto, Canada; ${ }^{3}$ University of Toronto, Department of Laboratory Medicine and Pathobiology, Toronto, Canada; ${ }^{4}$ Memorial University, Faculty of Medicine, St. John's, Canada

Background: Approximately $30 \%$ of psoriasis patients develop psoriatic arthritis (PsA), typically within 10 years of psoriasis onset. A large proportion of individuals with PsA remain undiagnosed. Epigenetics is potentially a major mechanism through which environmental factors influence PsA risk. An understanding of how the epigenome changes during the transition to PsA could yield predictive biomarkers and facilitate PsA diagnosis. We hypothesize that epigenetic deregulation at the level of DNA methylation occurs early in PsA pathogenesis, prior to overt clinical symptoms, and epigenetic marks can be used as biomarkers for disease prediction.

Objectives: To discover predictive biomarkers of PsA and gain an understanding of the pathogenesis of PsA by characterizing the epigenomic landscape of psoriasis patients who later developed PsA (converters) and comparing it to psoriasis patients who did not develop PsA (non-converters).

Methods: We performed an epigenome-wide comparison of DNA methylation in baseline whole blood samples from psoriasis converters $(n=60)$ and non-converters $(n=60)$ from a longitudinal cohort. Converters and non-converters were matched for age, sex, psoriasis duration, and duration of follow-up. DNA was analyzed on Human MethylationEPIC BeadChips using the ChAMP package. Cell type heterogeneity was corrected using RefbaseEWAS. Differentially methylated probes and regions were identified using limma and DMRcate, respectively. The FEM package was used to infer differentially methylated gene modules within a protein-protein interaction network.

Results: Converter baseline samples were collected a median of 4.2 (interquartile range 1.9-6.3) years prior to the onset of PsA, while non-converters samples were collected a median of $4.3(1.2-7.3)$ years prior to the most recent clinic visit. The RefbaseEWAS method estimated that converters had slightly higher proportions of CD4+ T cells and granulocytes compared to non-converters, however the differences were not statistically significant. After adjustment for cell type heterogeneity, 68 individual $\mathrm{CpG}$ sites were found to be differentially methylated between converters and non-converters (FDR<0.05). Differentially methylated regions (DMRs) containing at least 4 significant $\mathrm{CpGs}$ were identified in genes such as $F B X O 27$ (beta fold change $[F C]=0.06$, region-wise adjusted $p=4.1 \times 10^{-4}$ ), a ubiquitin ligase involved in lysosomal degradation, $R C A N 1\left(\mathrm{FC}=0.05, \mathrm{p}=5.2 \times 10^{-3}\right)$, a protein which inhibits calcineurin-dependent signaling pathways and is involved in bone homeostasis, and PMAIP1 ( $\left.\mathrm{FC}=0.04, \mathrm{p}=6.71 \times 10^{-3}\right)$, which encodes the NOXA protein involved in mediating apoptosis of activated B cells. Several significant $\mathrm{CpG}$ sites mapped to protein-protein interaction subnetworks involved in Th17 differentiation (IRF4 and MAF), TNF alpha signaling (IKBKE, REL, MAVS, 
TRAF7, MAFB), and Toll-like receptor signaling (TLR1, IRAK2 MYD88, TOLLIP, TICAM1, TRAM1).

Conclusion: Changes in individual CpGs, DMRs, and inflammatory pathways were detected in baseline samples of psoriasis converters compared to non-converters. These preliminary data support our hypothesis that DNA methylation changes occur early in PsA pathogenesis and can potentially serve as prognostic biomarkers of future onset of arthritis in psoriasis patients.

Disclosure of Interests: Remy Pollock: None declared, Rohan Machhar: None declared, Vinod Chandran: None declared, Dafna D Gladman Grant/research support from: AbbVie, Amgen, Celgene, Lilly, Novartis, Pfizer, and UCB, Consultant for: AbbVie, Amgen, BMS, Celgene, Galapagos, Gilead, Janssen, Lilly, Novartis, Pfizer, and UCB

DOI: 10.1136/annrheumdis-2019-eular.4185

\section{THURSDAY, 13 JUNE 2019}

\section{Adults are just grown up children! Discuss}

\section{OP0204 EMAPALUMAB, AN INTERFERON GAMMA (IFN-Y)- BLOCKING MONOCLONAL ANTIBODY, IN PATIENTS WITH MACROPHAGE ACTIVATION SYNDROME (MAS) COMPLICATING SYSTEMIC JUVENILE IDIOPATHIC ARTHRITIS (SJIA)}

Fabrizio De Benedetti ${ }^{1}$, Paul Brogan ${ }^{2}$, Alexei Grom ${ }^{3}$, Pierre Quartier ${ }^{4}$, Rayfel Schneider ${ }^{5}$, Kathy De Graaf ${ }^{6}$, Philippe Jacqmin ${ }^{7}$, Maria Ballabio ${ }^{6}$, Cristina de Min $6 .{ }^{1} I R C C S$ Ospedale Pediatrico Bambino Gesù, Rheumatology, Rome, Italy ${ }^{2}$ UCL Institute of Child Health, and Great Ormond Street Hospital NHS Foundation Trust, Section Head: Infection, Immunology, and Rheumatology, London, United Kingdom; ${ }^{3}$ Cincinnati Children's Hospital, Division of Rheumatology, Cincinnati, United States of America; ${ }^{4}$ Hôpital Necker, Service de Génétique clinique Génétique Moléculaire, Paris, France; ${ }^{5}$ The Hospital for Sick Children, Department of Paediatrics, Division of Rheumatology, Toronto, Canada; ${ }^{6}$ Novimmune SA, Basel, Switzerland; ${ }^{7}$ MnS Modelling and Simulation, Dinant, Belgium

Background: MAS is a severe complication of rheumatic diseases, most frequently SJIA, caused by excessive activation and expansion of $T$ cells and macrophages. It is characterized by fever, hepatosplenomegaly, liver dysfunction, cytopenias, coagulation abnormalities and hyperferritinemia, possibly progressing to multiple organ failure and death. MAS is categorized as a secondary form of HLH. A vast body of evidence points to uncontrolled overproduction of IFN $\gamma$ as a major driver of hyperinflammation and hypercytokinemia in MAS and HLH. Emapalumab has been shown to effectively control disease activity in patients with primary HLH.

Objectives: To assess the pharmacokinetics (PK), efficacy and safety of intravenous (IV) emapalumab in patients with MAS and confirm the proposed dose regimen.

Methods: This is a pilot open-label single arm international study (NCT03311854). Patients had to have MAS (defined according to the 2016 ACR/EULAR classification criteria), on a background of confirmed, or high presumption of, SJIA, and inadequate response to high-dose IV glucocorticoids. Emapalumab initial dose was 6 $\mathrm{mg} / \mathrm{kg}$ and treatment was continued at $3 \mathrm{mg} / \mathrm{kg}$, twice weekly for a total of 4 weeks or less upon achievement of complete response. Serum concentrations of emapalumab, IFN $\gamma$-induced chemokine CXCL9 and SIL2R were measured. Safety assessments included adverse events (AEs) and laboratory abnormalities. Efficacy was defined as complete response by week 8 , i.e. absence of MAS clinical signs plus white blood cell and platelet counts above lower limit of normal, LDH, AST/ ALT $<1.5 x$ upper limit of normal, fibrinogen $>100 \mathrm{mg} / \mathrm{dL}$, and ferritin decreased by $\geq 80 \%$ or to $<2000 \mathrm{ng} / \mathrm{mL}$, whichever was lower. Twin protocols are in place to recruit 5 patients each in Europe and North America (trial not started yet). We report data on the 6 patients recruited in the European protocol.

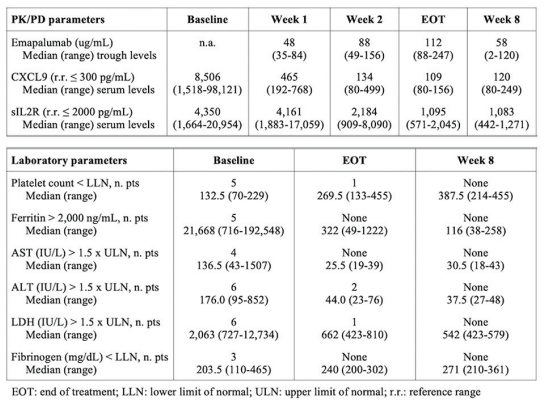

Results: Six patients (5 females, median age 11 years, range: 2-25 years) received emapalumab according to the prescribed dose regimen. Treatment duration ranged from 3 (2 patients) to 4 weeks. Prior to emapalumab, all patients failed methylprednisolone pulses, in 2 patients plus cyclosporine $A(C s A)$ and in 2 patients plus CsA and anakinra. The achieved emapalumab concentrations led to rapid neutralization of IFN $\gamma$ as indicated by CXCL9 levels and subsequent deactivation of $T$ cells as indicated by sIL2R levels. In all patients, complete response was achieved by week 8 , in 4 patients by end of treatment (Table). Systemic glucorticoids were weaned in all patients. Emapalumab infusions were well tolerated and none of the patients discontinued emapalumab. A CMV reactivation was reported by investigator as a serious event possibly related to emapalumab, but resolved completely with treatment.

Conclusion: Emapalumab administration with the tested dosing regimen led to rapid neutralization of IFN $\gamma$ as shown by normalization of CXCL9, associated with evidence of decreased T cell activation. In all patients, emapalumab treatment was effective in controlling MAS with a favourable safety profile.

\section{REFERENCE:}

[1] Ravelli A, et al. Ann Rheum Dis. 2016;75:481-9.

Disclosure of Interests: Fabrizio De Benedetti Grant/research support from: Abbvie, SOBI, Novimmune, Roche, Novartis, Sanofi, Pfizer, Paul Brogan Grant/ research support from: SOBI, Novartis, Roche, Novimmune, Chemocentryx, Consultant for: Roche, SOBI, Speakers bureau: SOBI, Roche, Novartis, UCB, Alexei Grom Grant/research support from: Novartis, AB2Bio, Novimmune, Consultant for: Novartis, Pierre Quartier Consultant for: AbbVie, Chugai-Roche, lilly, Novartis, Novimmune, Sanofi, and SOBI, Consultant for: AbbVie, Chugai-Roche, Lilly, Novartis, Novimmune, Sanofi, and SOBI, Speakers bureau: AbbVie, BMS, Chugai-Roche, Novartis, Pfizer, and SOBI, Speakers bureau: AbbVie, BMS, ChugaiRoche, Novartis, Pfizer, and SOBI, Rayfel Schneider Grant/research support from: Roche, Novartis, SOBI, Novimmune, Pfizer, Consultant for: Novimmune, SOBI, Novartis, Kathy De Graaf Consultant for: Novimmune, Employee of: Novimmune, Philippe Jacqmin Consultant for: I am a paid consultant in pharmacology for Novimmune, Maria Ballabio Shareholder of: Novimmune, Employee of: Novim mune, Cristina de Min Shareholder of: Novimmune, Employee of: Novimmune DOI: 10.1136/annrheumdis-2019-eular.3341

\section{OP0205 LIVE ATTENUATED VACCINES IN PEDIATRIC RHEUMATIC DISEASES ARE SAFE: MULTICENTER, RETROSPECTIVE DATA COLLECTION}

Yosef Uziel $^{1}$, Veronica Moshe Bergonzo ${ }^{2}$, Beata Onozo ${ }^{3}$, Andrea Kulcsar ${ }^{4}$, Diana Tróbert-Sipos ${ }^{4}$, Jonathan Akikusa ${ }^{5}$, Gecilmara Salviato Pileggi ${ }^{6}$, Despoina Maritsi ${ }^{7}$, Ozgur Kasapcopur ${ }^{8}$, Roubini Smerla ${ }^{9}$, Donato Rigante ${ }^{10}$, Erato Atasali ${ }^{11}$, Mariana Rodrigues ${ }^{12}$, Balahan Makay ${ }^{13}$, Nico Wulffraat ${ }^{14}$, Natasa Toplak ${ }^{15}$, PReS working party of Vaccination -Study group. ${ }^{1}$ Meir Medical Center, Kfar Saba, Israel; ${ }^{1}$ Meir Medical Center, Kfar Saba, Israel; ${ }^{3}$ Central Hospital of Borsod-Abauj-Zemplen, Miskolc, Hungary; ${ }^{4}$ South-Pest Central Hospital, Budapest, Hungary; ${ }^{5}$ The Royal Children's Hospital, Melbourne, Australia; ${ }^{6}$ Medical School of Barretos, Sao Paulo, Brazil; ${ }^{7}$ Athens Medical School, Athens, Greece; ${ }^{8}$ Cerrahpasa Medical School, Istanbul, Turkey; ${ }^{9}$ University of Athens, Athens, Greece; ${ }^{10}$ Fondazione Policlinico Universitario, Rome, Italy; ${ }^{11}$ Attikon University Hospital, Athens, Greece; ${ }^{12}$ São João University Hospital, Porto, Portugal; ${ }^{13}$ Behcet Uz Children's Hospital, Izmir, Turkey; ${ }^{14}$ Wilhelmina Kinderziekenhuis UMC Utrecht, Utrecht, Netherlands; ${ }^{15}$ University Children's Hospital Ljubljana, Ljubljana, Slovenia

Background: Common practice is to withhold vaccination with live-attenuated vaccines in patients with rheumatic diseases on high-dose DMARDs, glucocorticosteroids or biological agents, due to limited safety data, and the (theoretical) risk of introducing an infectious disease to the patient. Evidence for this approach is low. We collected data from pediatric rheumatologists who vaccinate these patients, to obtain additional safety data, which might update and revise this approach.

Objectives: To collect retrospective data in patients with JIA and other diseases who received live booster MMR or MMRV while on DMARDs, glucocorticosteroids or biological agents.

Methods: Data from 13 pediatric rheumatology centers in 10 countries were collected.

Results: 234 patients were reported; mean age $5 \pm 2.7,70 \%$ girls. 206 had JIA; $46 \%$ oligoarticular, $36 \%$ polyarticular, $8 \%$ systemic, $5 \%$ SPA types, $5 \%$ JIA and uveitis. $48 \%$ of $\mathrm{JIA}$ patients were in remission on medication. Disease activity was low in $38 \%$, high in $2 \%$, moderate in $7 \%$; 11 patients had juvenile dermatomyositis, 3 systemic and 2 localized scleroderma, 4 isolated idiopathic uveitis, 1 CINCA syndrome, $1 \mathrm{MKD}$, and $1 \mathrm{FMF}$.

110 patients had MMR/V booster while on MTX; 3 reported mild side-effects of local skin reaction and pain, none had disease flare. 76 had booster while on MTX + anti-TNF; 7 reported mild and transient adverse events of local skin reaction, fever and URTI. 39 had booster while on anti-TNF alone; 1 reported fever. 3 had 